\title{
ARONSZAJN'S THEOREM FOR SOME NONLINEAR DIRICHLET PROBLEMS WITH UNBOUNDED NONLINEARITIES
}

\author{
by JUAN J. NIETO
}

(Received 20th May 1986)

\section{Introduction}

We consider the Dirichlet problem

$$
u^{\prime \prime}+u+g(u)=h(x) \quad x \in(0, \pi), \quad u(0)=u(\pi)=0
$$

where $g$ is continuous and $h \in L^{2}(0, \pi)$.

By integrating (1) we see that a necessary condition for (1) to have a solution is that

$$
\omega=\omega(h)=\int_{0}^{\pi} h(x) \sin x d x / \int_{0}^{\pi} \sin x d x \in \overline{\text { Range } g}
$$

If $g$ is such that

$$
g(\infty) \leqq g(u) \leqq g(-\infty) \quad \text { for every } u \in \mathbb{R}
$$

then it is well known $[10,11]$ that a sufficient condition for the existence of solutions to (1) is that

$$
\omega \in \operatorname{Int}(\text { Range } g \text { ). }
$$

On the other hand, if $g$ satisfies

$$
g(-\infty) \leqq g(u) \leqq g(\infty) \quad \text { for every } u \in \mathbb{R},
$$

then a restriction on $g$ is needed. Indeed, for $g(u)=3 u$ and $h(x)=\sin 2 x$, problem (1) has no solution. Thus, we shall consider the following hypotheses

there exist constants $\gamma, C$ such that $C>0, \gamma \in[0,3)$, and $|g(u)| \leqq \gamma|u|+C$ for every $u \in \mathbb{R}$,

$$
g \text { is a nondecreasing function. }
$$


Ahmad proved in [1] that (1) has at least one solution provided that (6) holds and

$$
\overline{g(-\infty)} \int_{0}^{\pi} \sin x d x<\int_{0}^{\pi} h(x) \sin x d x<\underline{g(\infty)} \int_{0}^{\pi} \sin x d x
$$

where

$$
\overline{g(-\infty)}=\limsup _{u \rightarrow-\infty} g(u) \quad \text { and } \quad \underline{g(\infty)}=\liminf _{u \rightarrow \infty} g(u) .
$$

In section 2, we study the case when equality does occur in (8) (Theorem 1(c)). Then, we show that uniqueness does not occur in general even if (4) and (7) are satisfied. However, if $g$ is Lipschitz continuous and the Lipschitz constant "stays away" from the nearest eigenvalue uniqueness occurs (Theorem 2). Thus, we have that if there exists a non-negative constant $k$ with $k<3$ and

$$
|g(u)-g(v)| \leqq k|u-v|, \quad \text { for every } u, v, \in \mathbb{R}
$$

and $g$ is strictly increasing, then any solution of (1) is unique. The proof of this result is standard, but we give it for the sake of completeness.

In Section 3 we prove our main result (Theorem 4): If (4), (7) and (9) hold, then the set of solutions of $(1)$ in $L^{2}(0, \pi)$ is homeomorphic to the intersection of a decreasing sequence of compact absolute retracts. Following Aronszajn [2] we will call such a set an $R_{\delta}$. It is known that an $R_{\delta}$ is acyclic and, in particular, it is nonempty, compact and connected. Note that there are compact and connected sets which cannot be continuous images of $R_{\delta}$ 's $[12,13]$. We shall denote the solution set of (1) by $S(h)$.

Recently, Ballotti proved in [3] that the solution set for a parabolic partial differential equation is an $R_{\delta}$. Similarly, Górniewicz and Pruszko [6] and De Blasi and Myjäk [5] showed that the set of all solutions of a Darboux problem for a partial differential equation of hyperbolic type is an $R_{\boldsymbol{\delta}}$.

In our last section we present some examples in order to bring out the fact that the set of solutions is not an $R_{\delta}$ in general in the following two cases:

(i) $g$ satisfies (5) instead of (7)

(ii) $\omega \in$ Bdry (Range $g$ ).

In the sequel and for $p \in[1, \infty]$ we denote by ||$_{p}$ the usual norm in $L^{p}(0, \pi)$. For $R>0$, $\bar{B}_{p}(0, R)=\left\{u \in L^{p}(0, \pi):|u|_{p} \leqq R\right\}$. If $p=2, \quad u, v \in L^{2}(0, \pi)$ we write $\|u\|=|u|_{2}, \quad(u, v)=$ $\int_{0}^{\pi} u(x) v(x) d x$, and for $R>0, \bar{B}(0, R)=\bar{B}_{2}(0, R)$.

\section{Existence of solutions}

We have the following existence result.

Theorem 1. Under assumptions (0) and (7), we have:

(a) $\omega \in \operatorname{Int}($ Range $g$ ) is a sufficient condition for (I) to have a solution. 
(b) $\omega \in \overline{\text { Range } g}$ is a necessary condition for (1) to have a solution.

(c) If $\omega \in \mathrm{Bdry}$ (Range $g$ ), then (l) has a solution if and only if $g(0)=0$.

Proof. Part (a) is an immediate consequence of [1]. Part (b) follows by integrating (1), and (c) can be proved as in [9, Th. 2] (see also [10]).

We remark that in the case where $g$ is strictly decreasing, uniqueness is trivial since

$$
\left(u^{\prime \prime}+u+g(u)-v^{\prime \prime}-v-g(v), u-v\right)<0
$$

for every $u, v \in E$ with $u \neq v$. However, this is not true for $g$ non-decreasing. Indeed, for $h \equiv 0$ and $g$ strictly increasing with $g(u)=3 u$ in a neighbourhood of $u=0$, we have that $a \sin x$, with $a$ sufficiently small, are solutions of (1). We note that the non-uniqueness is due to the presence of an eigenvalue $(\lambda=3)$ of the problem

$$
u^{\prime \prime}+u+\lambda u=0, \quad u(0)=u(\pi)=0 .
$$

Note that the eigenvalues of $(10)$ are $\lambda_{i}=i^{2}-1, i=1,2, \ldots$. Nevertheless, we have the following uniqueness result.

Theorem 2. If $g$ is strictly increasing and (4) and (9) hold with $k<3$, then any solution of $(I)$ is unique.

Proof. Let $E=L^{2}(0, \pi)$ and define the operator $L: D(L) \subset E \rightarrow E$ by $L u=u^{\prime \prime}+u^{\prime \prime}$ where $D(L)=\left\{u \in H^{2}(0, \pi): u(0)=u(\pi)=0\right\}$. Let $N: E \rightarrow E$ be the Nemytskii map associated with the nonlinear part of (1), that is, $N u=h-g(u)$. Thus, (1) is equivalent to the operator equation $L u=N u$. Now, we take $c=\left(\lambda_{1}+\lambda_{2}\right) / 2=3 / 2$ (see [4, p. 116]). Thus, the operator $L+c I$ is invertible and $\left\|(L+c l)^{-1}\right\| \leqq c^{-1}$. On the other hand, for every $x, y \in \mathbb{R}$ we have that $|g(y)-g(x)+c(x-y)| \leqq c|x-y|$, with strict inequality when $x \neq y$ since $g$ is strictly decreasing and (9) holds with $k<3$. Therefore, $\|N u-N v+c(u-v)\| \leqq c\|u-v\|$ for every $u, v \in E$. Moreover, if $u \neq v$ in a set of positive measure, then $\|N u-N v+c(u-v)\|<c\|u-v\|$. Now, if $u, v \in E$ are two solutions then

$$
\begin{gathered}
(L+c I)^{-1}[N u-N v+c(u-v)]=u-v \quad \text { and } \\
\|u-v\| \leqq c^{-1}\|N u-N v+c(u-v)\|
\end{gathered}
$$

which implies that $u=v$. This completes the proof of the theorem.

\section{Aronszajn's theorem}

We shall use the following result due to Aronszajn [2].

Theorem 3. Let $K$ be a closed, convex and bounded set is a Banach space $E$. Let $T: E \rightarrow E$ be compact such that

(a) $T(K) \subset K$ 
(b) For every $\varepsilon>0$, there exists $T_{\varepsilon}: E \rightarrow E$ compact with $\left\|T_{\varepsilon}(u)-T(u)\right\|<\varepsilon$ for every $u \in K$

(c) There exists $\rho>0$ such that for every $\varepsilon>0, I-T_{\varepsilon}$ maps $K$ bijectively onto a set containing $\bar{B}(0, \rho)=\{u \in E:\|u\| \leqq \rho\}$.

Then the set of fixed points of $T, F(T)=\{u \in E: T(u)=u\}$ is an $R_{\sigma}$.

Now we are in a position to prove our main result.

Theorem 4. Suppose that (4), (7) and (9) hold with $k<3$. Then the set of solutions of $(1)$ is an $R_{\delta}$.

Proof. Let $\xi(x)=(\pi / 2) \overline{\sin x}, x \in[0, \pi]$ and define the projection $P: E \rightarrow E$ by $P u=$ $(u, \xi) \xi$. Thus, $P E=E_{0}=\operatorname{Ker} L$ and $E=E_{0} \oplus E_{1}, E_{1}=(I-P) E$. The partial inverse of $L$ is $H: E_{1} \rightarrow E_{1}$ where $H v=u$ iff $L u=v, u \in E_{1}$. It is well known [4] that solutions of $L u=N u$ are precisely the fixed points of the operator $R: E \rightarrow E, R u=P u+H(I-P) N u+$ PNu.

On the other hand, we know [1] that there exists $R>0$ such that $|u|_{\infty} \leqq R$ for any $u \in S(h)$. Choose $A<-R, B>R$ such that $g(A)<\omega(h)<g(B)$.

Consider the following modified problem

$$
u^{\prime \prime}+u+G(u)=h(x), \quad x \in(0, \pi), \quad u(0)=u(\pi)=0
$$

where

$$
G(u)= \begin{cases}g(A), & u<A \\ g(u), & A \leqq u \leqq B \\ g(B), & u>B .\end{cases}
$$

Now, let $M: E \rightarrow E, M u=h-G(u)$ so that (11) is equivalent to $L u=M u$. Any solution of (1) is also a solution of (11) and taking into account Theorem 1(a) we have that (1) is solvable. Hence, if we show that the set of solutions of (11), denoted by $\tilde{S}(h)$, is an $R_{\delta}$, we can conclude that

$$
S(h)=\widetilde{S}(h) \cap \bar{B}_{\infty}(0, R)
$$

is an $R_{\boldsymbol{\delta}}$.

We note that $\tilde{S}(h)$ is bounded since $G$ is bounded and $g(A)<\omega(h)<g(B)$. Let $s$ be such that $\|u\| \leqq s$ for every $u \in \tilde{S}(h)$. Define the retraction $r: E \rightarrow \bar{B}(0, s)$ by $r u=u$ for $\|u\| \leqq s$, $r u=(s /\|u\|) u$ for $\|u\|>s$. In order to show that $\bar{S}(h)$ is an $R_{\delta}$ we consider the operator $T: E \rightarrow E$ defined by $T u=P r u+H(I-P) M u+P M u$. Note that

$$
\widetilde{S}(h)=F(T) \cap \bar{B}(0, R) \text {. }
$$

We shall show that $F(T)$ is an $R_{\delta}$ by using Aronszajn's theorem. Let $J$ be such that $\|M u\| \leqq J$ for $u \in E$ and set $c=\|H(I-P)\| \cdot J$. 
Let $K=\bar{B}(0, t)$ where $t=s+c+J+\rho, \rho>0$. For $u \in K,\|T u\| \leqq s+c+J<t$ which shows that $T(K) \subset K$.

Now, let $\phi: \mathbb{R}^{+} \rightarrow \mathbb{R}^{+}$be a continuous and strictly increasing function such that $\phi(\varepsilon)>0$ for $\varepsilon>0$ and $\phi(\varepsilon)<\min \{\pi /(\pi \sqrt{2} \pi(\|H(I-P)\|+1)), 3-K\}$. Define $M_{\varepsilon}: E \rightarrow E$ by $M_{\varepsilon}(u)=M u-\phi(\varepsilon) \operatorname{Arctan} u$, and $T_{\varepsilon}(u)=P r u+H(I-P) M_{\varepsilon}(u)+P M_{\varepsilon}(u)$. Thus, for $u \in K$ we get $\left\|T_{\varepsilon}(u)-T(u)\right\| \leqq\|H(I-P)\| \cdot \phi(\varepsilon) \cdot\|\operatorname{Arctan} u\|+\phi(\varepsilon) \cdot\|\operatorname{Arctan} u\|<\varepsilon$. Hence, (b) of Theorem 3 is satisfied.

To show (c) we shall prove that $I-T_{\varepsilon}$ is one-to-one in $K$ and $\bar{B}(0, \rho) \subset\left(I-T_{2}\right) K$. Indeed, let $u, v \in K$ such that $\left(I-T_{e}\right) u=\left(I-T_{e}\right) v$. Thus, $u-v=T_{e} u-T_{e} v$. Therefore, $u-v \in D(L)$ and $L(u-v)=M_{\varepsilon} u-M_{\varepsilon} v=M u-M v-\phi(\varepsilon)$ [Arctan $\left.u-\operatorname{Arctan} v\right]$. The function $u \rightarrow G(u)+\phi(\varepsilon) \operatorname{Arctan} u$ is strictly increasing and Lipschitz continuous with Lipschitz constant $k+\phi(\varepsilon)<3$. By Theorem 2 we can conclude that $u=v$.

Now, for $w \in \bar{B}(0, \rho)$, define the operator $A_{\varepsilon}: E \rightarrow E, A_{\varepsilon}(u)=T_{\varepsilon}(u)+w$. For $u \in K$, $\left\|A_{\varepsilon}(u)\right\| \leqq\left\|T_{\varepsilon}(u)\right\|+\rho<t$. In consequence, $A_{\varepsilon}(K) \subset K$ and by Schauder fixed point theorem $A_{\varepsilon}$ has a fixed point $u \in K$ which is precisely the solution of $\left(I-T_{\varepsilon}\right) u=w$. This shows (c) of Aronszajn's theorem and that the set of fixed points of $T$ is an $R_{\delta}$.

From (12) and (13) we can conclude that $S(h)$ is an $R_{\delta}$ since, as is well known, any convex subset of a Banach space is an absolute retract.

\section{Counterexamples}

In this section we show with some examples that Theorem 4 is as sharp as possible in the sense that the solution set is not an $R_{\delta}$ if we have either

(i) (5) instead of (7), or

(ii) $\omega \in$ Bdry (Range $g$ ) instead of (4)

even if (9) holds with $k<3$.

Example 1. Let $h \equiv 0$ and

$$
g(u)=\left\{\begin{array}{rc}
-1, & u<-1 \\
u, & -1<u<0 \\
0, & u>0 .
\end{array}\right.
$$

Clearly $g$ is non-decreasing, $g(-\infty)=-1$, and $g(\infty)=0$. On the other hand, $\omega(h)=0$ and if $u$ is a solution of (1), then

$$
\int_{0}^{\pi} g(u(x)) \sin x d x=0
$$

This implies that $u(x) \geqq 0$ for every $x \in[0, \pi]$ since $g \leqq 0$. Therefore, the set of solutions is given by $\{\alpha \sin x: \alpha \geqq 0\}$ which is not bounded. Hence, the solution set is not an $R_{\delta}$. Note that the solution set is connected in this case. 
Remark. If $\omega \in$ Bdry(Range $g$ ) and $g$ is strictly increasing, then (1) has no solution by Theorem 1(c). Hence, the solution set would be empty and it cannot be an $R_{\delta}$.

Example 2. Consider the problem

$$
u^{\prime \prime}+u+g(u)=-\frac{1}{2}, \quad u(0)=u(\pi)=0
$$

where

$$
g(u)=\left\{\begin{array}{cl}
u, & u<0 \\
-u, & 0 \leqq u \leqq 1 \\
u-2, & u>1 .
\end{array}\right.
$$

Thus, $\omega=-\frac{1}{2}$ and (4) holds. It is easy to see that $g$ satisfies (5) and (9) with $k<3$. In fact, $k=1$.

We shall show that $S(h)$ is not connected and consequently it is not an $R_{\delta}$.

If $u$ is a solution of (14) such that $0 \leqq u \leqq 1$ in $[0, \pi]$, then $u$ is a solution of the linear problem $u^{\prime \prime}=-\frac{1}{2}, u(0)=u(\pi)=0$. Thus, $\alpha(t)=-\frac{1}{4} t(t-\pi)$ is a solution of (14) since $0 \leqq \alpha \leqq 1$ in $[0, \pi]$. On the other hand, if $u$ is a solution with $u \leqq 0$, then $u$ satisfies the linear problem $u^{\prime \prime}+2 u=-\frac{1}{2}, u(0)=u(\pi)=0$, which has a unique solution given by $\beta(t)=-\frac{1}{4}$ $+\lambda \sin \sqrt{2} t+\mu \cos \sqrt{2} t$ where $\lambda=(1-\cos \sqrt{2} \pi) / 4 \sin \sqrt{2} \pi, \mu=\frac{1}{4}$. Note that $\lambda<0$ and set $m_{0}=\beta^{\prime}(0)=\lambda \sqrt{2}$.

Now, for $m \in \mathbb{R}$, consider the initial value problem

$$
u^{\prime \prime}+2 u=-\frac{1}{2}, \quad u(0)=0, \quad u^{\prime}(0)=m
$$

which has a unique solution $u_{m}(t)=-\frac{1}{4}+\lambda_{m} \sin \sqrt{2} t+\mu_{m} \cos \sqrt{2} t$ with $\lambda_{m}=2^{-1 / 2} \cdot m$ and $\mu_{m}=\frac{1}{4}$.

We prove below that

$$
u_{m}(t) \leqq 0 \quad \text { for every } m \in\left[m_{0}, 0\right], \quad t \in[0, \pi] .
$$

Note that $\beta=u_{m_{0}}$. Thus, $\beta$ is a solution to (14). If $m \in\left(m_{0}, 0\right]$, then $u_{m}(\pi) \leqq-\frac{1}{4}+$ $\lambda_{m} \sin \sqrt{2} \pi+\mu_{m} \cos \sqrt{2} \pi<0$. Hence, $u_{m}$ is not a solution of (14) for $m \in\left(m_{0}, 0\right]$.

On the other hand, the initial value problem $u^{\prime \prime}+u+g(u)=-\frac{1}{2}, u(0)=0, u^{\prime}(0)=m$, has a unique solution for any $m \in \mathbb{R}$. For $m \in\left[m_{0}, 0\right]$ such a unique solution is precisely $u_{m}$. Therefore, (14) has no solution $u$ with $u^{\prime}(0) \in\left(m_{0}, 0\right)$.

Let $c=m_{0} / 2$ and consider the open sets in $X=C^{1}[0, \pi]$.

$$
A=\left\{u \in X: u^{\prime}(0)>c\right\}, \quad B=\left\{u \in X: u^{\prime}(0)<c\right\} .
$$

Thus, $\alpha \in A \cap S(h), \beta \in B \cap S(h), A \cup B \supset S(h)$, and $A \cap B=\varnothing$ which means that $S(h)$ is not connected in $X$. Moreover, $S(h)$ is not connected in $E$. Indeed, the operator $R$ can 
be considered as a continuous map form $E$ to $X$, and $R(S(h))=S(h)$. This implies that $S(h)$ is not connected in $E$.

Proof of (15). For $t \in\left[0,2^{-1 / 2} \cdot \pi\right]$ we have that $\sin \sqrt{2} t \geqq 0$. Thus, $\lambda_{m} \sin \sqrt{2} t \leqq 0$ and $u_{m}(t) \leqq 0$. If $t \in\left[2^{-1 / 2} \cdot \pi, \pi\right]$, then $\cos \sqrt{2} \pi \geqq \cos \sqrt{2} t$ and $\sin \sqrt{2} \pi \leqq \sin \sqrt{2} t \leqq 0$. This implies that $u_{m}(t) \leqq-\frac{1}{4}+2^{-1 / 2} \cdot m \sin \sqrt{2} \pi+\frac{1}{4} \cos \sqrt{2} \pi \leqq 0$.

Acknowledgement. The author is thankful to the referee for helpful comments and suggestions.

\section{REFERENCES}

1. S. Ahmad, A resonance problem in which the nonlinearity may grow linearly, Proc. Amer. Math. Soc. 82 (1984), 381-384.

2. A. AronszasN, Le correspondant topologique de l'unicité dans le theorié des équations différentielles, Ann. Math. 43 (1942), 730-738.

3. M. E. BallortI, Aronszajn's theorem for a parabolic partial differential equation, Nonlinear Anal. 9 (1985), 1183-1187.

4. L. Cesari, Functional analysis, nonlinear differential equations and the alternative method, Nonlinear Functional Analysis and Differential Equations (L. Cesari, R. Kannan and J. Schur, Eds) (Marcel-Dekker, 1976), 1-197.

5. F. S. De Blasi and J. Myjak, On the structure of the set of solutions of the Darboux problem for hyperbolic equations, Proc. Edinburgh Math. Soc. 29 (1986), 7-14.

6. L. Gorniewicz and T. Pruszko, On the set of solutions of the Darboux problem for some hyperbolic problems, Bull. Acad. Polon. Sci. Sér. Sci. Math. Astronom. Phys. 38 (1980), 279-285.

7. J. K. Hale, Applications of Alternative Problerns (Lecture Notes of Brown University, Providence, R.I., 1971).

8. D. M. Hyman, On decreasing sequences of compact absolute retracts, Fund. Math. 64 (1969), 91-97.

9. R. Kannan, V. Laksmikantham and J. J. Nieto, Sufficient conditions for existence of solutions of nonlinear boundary value problems at resonance, Nonlinear Anal. 7 (1983), 1013-1020.

10. R. Kannan, J. J. Nieto and M. B. Ray, A class of nonlinear boundary value problems without Landesman-Lazer condition, J. Math. Anal. Appl. 105 (1985), 1-11.

11. E. M. Landesman and A. C. Lazer, Nonlinear perturbations of linear elliptic boundary value problems at resonance, J. Math. Mech. 19 (1970), 609-623.

12. C. C. Pugr, Funnel sections, J. Differential Equations 19 (1975), 270-295.

13. J. T. RogerTs, The shape of the cross-section of the solution funnel of an ordinary differential equation, Illinois J. Math. 21 (1977), 420-426.

Departamento De Analisis Matematico

Universidad De Santiago

SPAIN 